Voix et Images

voixetimages

\title{
Le titre du roman québécois des années 1940-1960
}

\section{Richard Berger}

Volume 7, numéro 1, automne 1981

\section{Adrien Thério}

URI : https://id.erudit.org/iderudit/200305ar

DOI : https://doi.org/10.7202/200305ar

Aller au sommaire du numéro

Éditeur(s)

Les Presses de l'Université du Québec

ISSN

0318-9201 (imprimé)

1705-933X (numérique)

Découvrir la revue

Citer cet article

Berger, R. (1981). Le titre du roman québécois des années 1940-1960. Voix et Images, 7(1), 79-94. https://doi.org/10.7202/200305ar d'utilisation que vous pouvez consulter en ligne.

https://apropos.erudit.org/fr/usagers/politique-dutilisation/ 


\title{
Le titre du roman québécois des années 1940-1960
}

\author{
par Richard Berger
}

«L'un annonce, l'autre explique»

Claude Duchet *

A l'idéologie de la propriété du texte, faisant de l'auteur l'unique propriétaire d'une matière signifiante, se double l'idéologie de l'identification: le titre. Le produit, le texte (ici, romanesque) ou son institutionnalisation, appelle la nécessité de s'identifier; donc, à la fois de se différencier et de s'annoncer, de se poser en tant que texte. Et comme il s'agit de livres sur un marché, on voit immédiatement dans le titre un nom propre, une marque du texte.

Par ailleurs, fait intéressant et révélateur à noter, plusieurs écrits du Moyen-Age et de la Renaissance étaient titrés sans que ne paraisse la signature de leur auteur, ce qui accusait, devant la griffe, l'importance de cette marque, du moins sur un certain plan. En effet, le livre demande à être re-connu (en son genre) et lu. Par conséquent, le titre assume deux fonctions: référentielle (donner le nom du livre) et conative (intéresser le lecteur au livre). "La page de titre est un support publicitaire». La situation de marché dans lequel se trouve le livre (ici le roman) fait du titre un message codé résultant "de la rencontre d'un énoncé romanesque et d'un énoncé publicitaire ${ }^{1}$ ». Ainsi, le titre peut se laisser investir, tout comme le nom de l'auteur, d'une valeur d'usage (fonction référentielle de l'ordre de la dénomination) aussi bien que d'une valeur d'échange (fonction référentielle de l'ordre d'une nomination en tant que valorisation).

Le titre est donc une/la marque du texte qu'il veut désigner. Il en est une inscription, un nom propre constituant un signe (presque un sujet). Or, dans le nom propre, contrairement au nom commun, «le sens s'institue par une convention individuelle ${ }^{2}$, la relation qui s'établit entre le référé et le signifiant étant d'ordre spécifique. Le nom commun «table» peut référer à toutes les tables; le nom propre "Félix" ne réfère, lui, qu'à la seule personne qui porte ce nom ${ }^{3}$.

\footnotetext{
* Duchet, 1973, p. 51.
} 
Toutefois, le problème de la référence du titre est un peu plus complexe. $S$ 'il nomme le texte qui suit, il en indique aussi un élément. Fonction économique: condensation/métaphore; déplacement/métonymie du texte. Méta-signe puisque «signe, il réfère à un ensemble de signes constituant la relation (I'histoire) comme sens, en en faisant attendre ou supposer l'intérêt". "A l'interrogation du titre, correspond le roman comme réplique ${ }^{4}$. De cette manière, le titre du roman désigne un manque à savoir, négativité, et pose de la sorte comme bénéfice la lecture du livre: positivité du texte. II a donc une fonction textuelle fortement marquée.

C'est dans cette logique textuelle du titre que je chercherai ici à rendre compte de son fonctionnement dans la production romanesque québécoise des années 1940 à 1960 à l'aide d'un recensement de plus de deux cent soixante titres-énoncés. Et comme le titre ne fait que «programmer au maximum la lecture ${ }^{5} \%$, on est en droit de s'attendre à déceler dans ce fonctionnement un mécanisme de réponse à l'horizon d'attente de l'époque ${ }^{6}$. En effet, de par ses fonctions doublement référentielle (livre et texte) et conative, il est légitime de supposer que le titre a une importance non mesurée encore dans la réception du texte qu'il désigne. Et c'est ce que je veux démontrer ici.

Pour ce faire, je tenterai d'établir une sémiotique du titre romanesque de la période couverte par une analyse à trois niveaux. D'abord, la syntaxe visera à exposer la codification du titre en termes relationnels de catégories. Ensuite, la sémantique verra a établir les réalisations du titre: la relation entre le sens et le référent pourra montrer un fonctionnement peut-être spécifique des valorisations textuelles de l'époque. La sémantique cherchera à établir deux types de rapport: rapport du titre au texte (type d'information que transmet le titre sur le texte) et rapport du titre aux autres titres ( "thèmes" repérables sur l'ensemble de la production de l'époque). Enfin, en dernière analyse, on tentera d'instituer une pragmatique du titre ${ }^{7}$ en essayant de justifier une relation entre le signe (titre) et ses utilisateurs (interprétants), tout en tenant compte de la fonction médiatrice que le titre maintient entre le texte et le lecteur.

\section{La syntaxe}

Si le titre permet d'annoncer le texte qui le suit, c'est qu'il met en syntagme une série d'éléments. Cette mise en contexte est marquée presque exclusivement (du moins pour la période concernée) par un style nominal. En tant qu'énoncé simple (ou simplifié, comme on le verra), le titre semble présenter une certaine a-grammaticalité (au sens chomskyien) provoquée par l'absence systématique de verbe. Or, selon Grivel, le défaut du verbe entraîne une distanciation par rapport au point de référence et permet d'accroître l'aptitude du titre à fonctionner comme signe dans la mesure où il gagne en équivocité ${ }^{8}$.

Pour sa part, Hoek croit en une «performativité" du titre. "Le titre fait ce qu'il dit: le titre $X$ réfère au texte nommé $X{ }^{9}$ ». En fait, selon lui, en structure 
profonde existerait une phrase performative de la forme: «Je déclare à toi que ce texte dit $X$ » de laquelle il ne reste, après transformations, en structure de surface que « $X$ ». De plus, la «performativité du titre contribue à sa «vraisemblabilisation" parce qu'elle présuppose que l'effet du titre ne sera pas nul ${ }^{10}$ \%. Ceci maintient évidemment l'idéologie de «l'effet sincère» du titre, effet que transgresse par ailleurs le Nouveau Roman.

Pour rendre compte de cette phrase performative, j'utiliserai (avec plus de respect que ne l'a fait Hoek) l'appareil chomskyien d'avant 1973 (c'est-à-dire celui présenté dans Aspects of Theory of Syntax) avec, pour exemple, un modèle fréquemment rencontré dans le corpus analysé:

Construction syntaxique d'un modèle fréquemment rencontré dans le corpus:
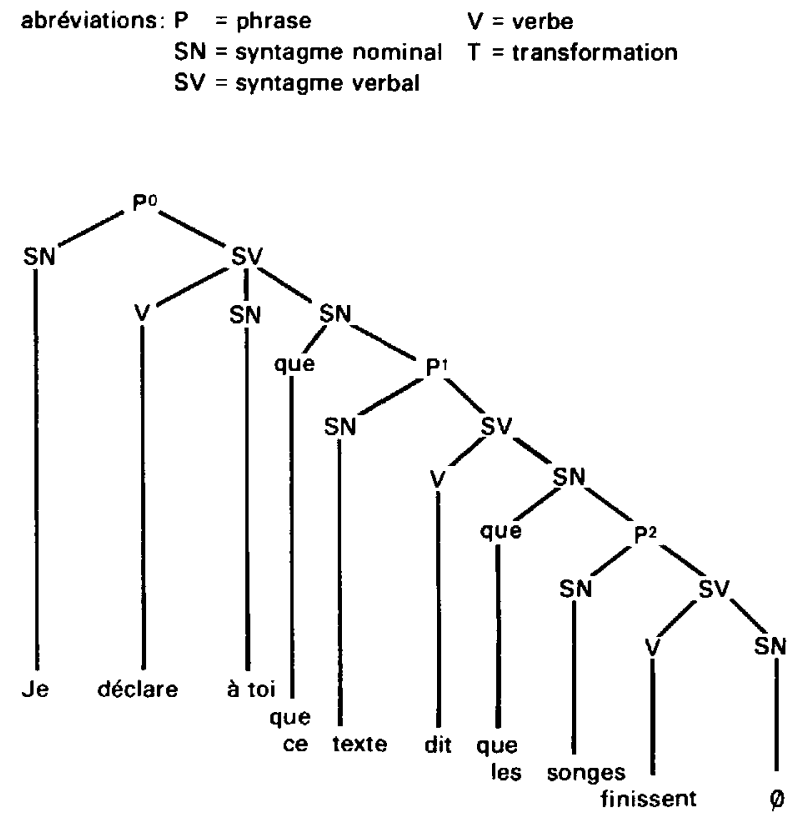

T d'effacement :
$\emptyset$
$\varnothing$
$\emptyset \emptyset \emptyset$
$\emptyset$ les songes finissent

$T$ de nominalisation

+ la fin de

T de déplacement: 
Evidemment, c'est $P^{2}$ qui contient l'essence (I) syntaxique du titre, la profondeur de son enchâssement indiquant probablement la rigidité de sa position quant à la manifestation en surface de la phrase performative. Puisque $P^{0}$ et $P^{1}$ sont entièrement effacées, parler de la syntaxe du titre, c'est parler de la syntaxe de $P^{2}$. C'est ce à quoi je m'en suis tenu pour les deux cents soixante-trois titres étudiés. Je n'en présenterai ici que la réduction des principaux modèles.

D'abord, on retrouve la phrase nominale (comportant également les expansions adjectivales) avec, par exemple: La Voix de l'âme (1944), Les Fous de lîle heureuse (1952) ou plus simplement La Louve (1952). Ce modèle, regroupant aussi bien les phrases nominalisées que les parataxes ou les simples syntagmes nominaux, constitue statistiquement soixante-sept titres, i.e. soixante-quatre pour cent du corpus. Ensuite, le nom propre, seul ou accompagné d'un syntagme nominal, représente vingt et un pour cent du corpus avec des titres comme Zriska, immigrante inconnue (1947) ou La Vie tourmentée de Michel Róbal (1946) ou encore Alexandre Chenevert (1954). Si l'on considère le nom propre comme une forme particulière de syntagme nominal, on obtient quatre-vingt cinq pour cent des titres produits essentiellement par un ou plusieurs SN. La quasi-absence de verbe est tout à fait confirmée, d'autant plus qu'un titre comme, par exemple, Le diab/e apparaît à St-Tristan (1952) aurait pu/dû se lire «L'apparition du diable à St-Tristan».

En termes de typologie des genres (concurrents de 1940 à 1950), il faut signaler que le roman historique (Les Opiniâtres, Nord-sud, L'Épopée tragique, La Fille du roy, etc.) utilise dans ses titres, en termes de tendance, exclusivement le premier modèle avec la présence assez marquée du féminin. De son côté, le roman social (Lemelin, Roy, Richard, Gélinas, etc.) use davantage du nom propre tandis que le roman psychologique (Charbonneau, Loranger, Giroux, Élie, etc., beaucoup plus nombreux car dominants dès 1950) est le plus conforme à la formule nominale et emploie plus souvent que tout autre genre le pluriel.

\section{La sémantique}

II s'agit maintenant de comprendre les relations existant non plus dans la syntagme du titre, mais entre lui et son référent. Or, le titre réfère toujours au texte qui le suit, au contexte ${ }^{11}$, fut-ce fragmentairement ${ }^{12}$. II ne reprend qu'un élément du contexte pour le montrer d'avance à l'attention du lecteur qui l'interprètera comme élément principal à mémoriser. Duchet parle d'anticipation et de mémorisation en distinguant le titre métaphorique (équivalent symbolique du texte) du titre métonymique (partie du texte pour le texte entier) ${ }^{13}$. Et Grivel prétend que le titre constitue *un complexe, une agglomération d'informations inachevées, un lieu d'indices ${ }^{15} \%$. C'est pourquoi il est possible de parler d'un manque à savoir, d'un point d'ancrage et/ou d'un point de relais (pour réutiliser une terminologie propre à Barthes ${ }^{15}$ ) par rapport au texte. 
J'ai donc examiné, pour le corpus concerné, quel(s) sens était(ent) mis en cuvre dans et par le titre. J'ai utilisé les grilles d'analyse de Grivel ${ }^{16}$ et de Hoek 17, ainsi que la notion de "thème". Cette façon de procéder a permis de relever certaines constantes sémiques du titre de l'époque. Le signe étant une représentation du rapport au réel, les articulations sémiques fournissent des axes sémantiqués à partir desquels on peut (tender $d^{\prime}$ ) établir des « substrats idéologiques » inhérents à l'ensemble du corpus. Du moins, c'était là l'intention visée, car il ne faut pas oublier: d'une part, que le titre a une double fonction référentielle, certes, mais aussi une fonction conative, ce qui peut influer sur la focalisation sémique qu'effectue le titre sur le contexte (la fonction publicitaire étant de surenchérir pour vendre); et, d'autre part, qu'il doit respecter certaines constructions codées, identifiant le genre (psychologique, social...), sinon le macro-genre (roman, poésie...) ${ }^{18}$. Les «substrats idéologiques" repérés ont pu, évidemment, servir de point de comparaison avec l'horizon d'attente des genres en concurrence à l'époque.

2.1 La méthode de Grivel est excessivement lourde pour les maigres résultats qu'elle permet d'obtenir. Sur un échantillon de quarante-cinq titres et en comparaison avec le corpus de Grivel, ce qui frappe l'attention, d'abord, c'est la presque absence de statut social accordé aux agents désignés dans/par le titre. Ni marques de noblesse, de bourgeoisie, de richesse ou d'état confessionnel. De plus, les marques d'état hiérarchique et d'état civil de l'agent sont plutôt rares (toujours en comparaison avec le corpus de Grivel). Même phénomène en ce qui concerne la qualification physique. Les indications temporelles et la localisation n'ont un caractère que général: il s'agit de «jour/nuit» ou de «refuge/maison» ou encore de «montagne/rue», etc. Ni date ou jour de semaine, ni toponymes. En revanche, les titres étudiés insistent davantage sur les noms propres, parfois les valeurs morales et sur les objets. Autres traits assez conformes au code romanesque valorisé à l'époque.

On peut retenir que l'absence (ou le flou) des traits sociaux et physiques des agents ainsi que des traits spatio-temporels précis n'est pas insignifiante et correspond à l'horizon d'attente. Car, en fait, comme il a été signalé ailleurs ${ }^{19}$, la production dominante de l'époque (roman psychologique) rejetait toute conception sociale du roman et optait pour l'idéologie personnaliste chrétienne (la problématique de cette production est l'universalisme; d'où l'absence de différenciations sociales, régionales et temporelles). Ce qui laisse supposer une certaine adéquation entre titre et réception (ici, je renvoie à la pragmatique).

2.2 Pour sa part, Hoek s'interroge sur le type d'informations que transmet le titre. II en distingue cinq: information actantielle, spatiale, temporelle, événementielle et objectale. Une sixième catégorie s'ajoute: le titre non fictionnel renvoyant davantage à un mode d'écriture qu'au texte lui-même (l'autobiographie, par exemple).

La classification des titres étudiés laisse entrevoir une distribution particulière, notamment en ce qui concerne l'information actantielle et 
événementielle. Voici les proportions obtenues (certains titres ont été classés dans plus d'un type, ce qui explique que le total des pourcentages dépasse 100):

a) information actantielle: 107/263: $41 \%$. C'est le nom propre qui est l'information la plus fréquente sur l'actant. Exemples: La Conscience de Pierre Laubier (1943), Marie Didace (1947), La Veuve (1955).

b) information spatiale: $41 / 263$ : $15,2 \%$. Le nom commun est de loin plus fréquent que le toponyme; information fort générale (chambre, montagne, etc.). Exemplès: La Terre du huitième (1942), La Vallée des blés d'or (1948), Carrefour des hasards (1959).

c) information temporelle: 19/263: $7,3 \%$. Information encore très générale. Exemples: Dans la nuit sombre (1942) ${ }^{20}$, Le Jour de I'Indien (1955), Et le septième jour (1958).

d) information événementielle: 66/263: 25,2\%. Qualification implicite de l'événement; donc, presque nécessairement un titre métaphorique ou très direct. Exemples: Impasse (1946), Le Poids du jour (1949), Le Gouffre a toujours soif (1953).

e) information objectale : $17 / 263: 6,5 \%$. Surtout des instruments. Exemples: De l'épée à la croix (1945), La Coupe vide (1948), Le Sabre d'Arlequin (1956).

f) titres non fictionnels: $5 / 263: 1,9 \%$. Genre étude de mœurs et biographie (Sa marottre, Etudes de moeurs canado-américaines, 1944) et (Quelle vie, biographie d'une canadienne-française, 1940).

g) non classées: 13/263: 4,9\%. Du genre: L'Tréelle (1944).

Schématiquement représentées, les proportions se lisent ainsi:

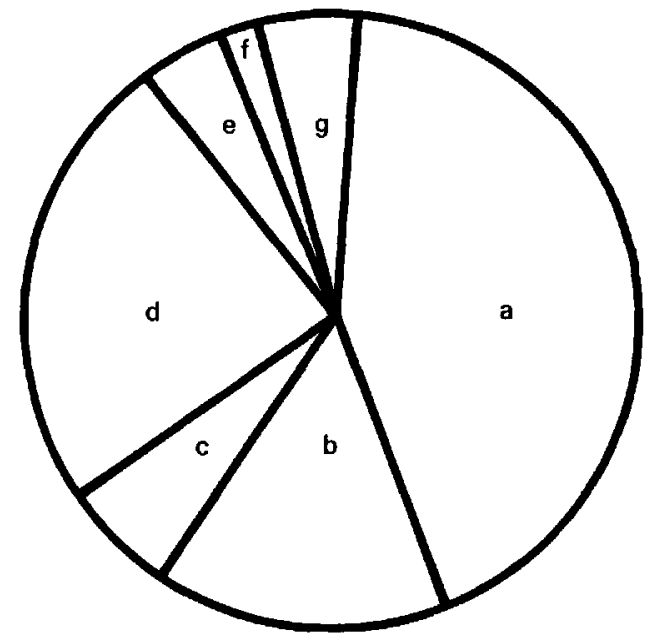


On voit donc que les titres mettent particulièrement l'accent sur l'information actantielle et l'information événementielle. II s'agit peut-être là d'une logique ou d'une contrainte du code titrologique; il faudrait vérifier sur d'autres corpus. Cependant, dans une perspective diachronique (année par année), j'ai constaté que les proportions dominantes fluctuent assez significativement. L'accent mis sur l'agent est beaucoup plus fréquent dans les titres de romans publiés entre 1944 et 1954 (pratiquement un titre sur deux). L'information événementielle, très fluctuante, maintient tout de même un caractère stable entre 1946 et 1952. Par contre, on peut pratiquement placer en rapport inverse les informations spatiale et temporelle (leurs sommets se situent avant 1942 et après 1952 pour la spatiale et 1956 pour la temporelle). Cette situation coïncide avec la période de cristallisation du genre "cas de conscience» (ou psychologique) qu'on a située vers $1950^{21}$.

2.3 Des valorisations d'un autre ordre sont décelables dans l'ensemble du corpus. En effet, des sèmes communs à plusieurs titres ont été repérés. constituant différents «axes thématiques». Ainsi, j'ai cherché à retracer les thèmes titrologiques de l'époque en définissant le thème comme catégorie sémantique présente (de façon redondante) dans l'étendue du corpus. Je ne peux prétendre avoir épuisé le réservoir sémantique de ces 263 titres, loin de là, mais, en me basant sur le critère de récurrence d'une même catégorie sémantique, j'ai sûrement relevé du corpus des thèmes importants. Cinq principaux «axes thématiques" mettent ainsi en position (souvent contraire) deux catégories sémantiques:

1. l'âme, l'esprit, la conscience

2. I'individu (I'unique)

3. I'amour (et le bonheur)

4. le destin

5. Ies lieux vs 1 ' le corps

vs 2' la collectivité (le pluriel)

vs 3' la haine (et le malheur)

vs 4' l'acte volontaire

vs $5^{\prime}$ le temps. 
Après avoir constitué un premier inventaire pour la période 1940-1960, j'en ai établi un second pour les années 1920-1940, avec, comme critère de sélection, les mêmes «axes thématiques». Après pondération des résultats (pour fins de comparaison équitables), j'ai pu représenter graphiquement des «aires de recouvrement sémantique» pour chacune des périodes:

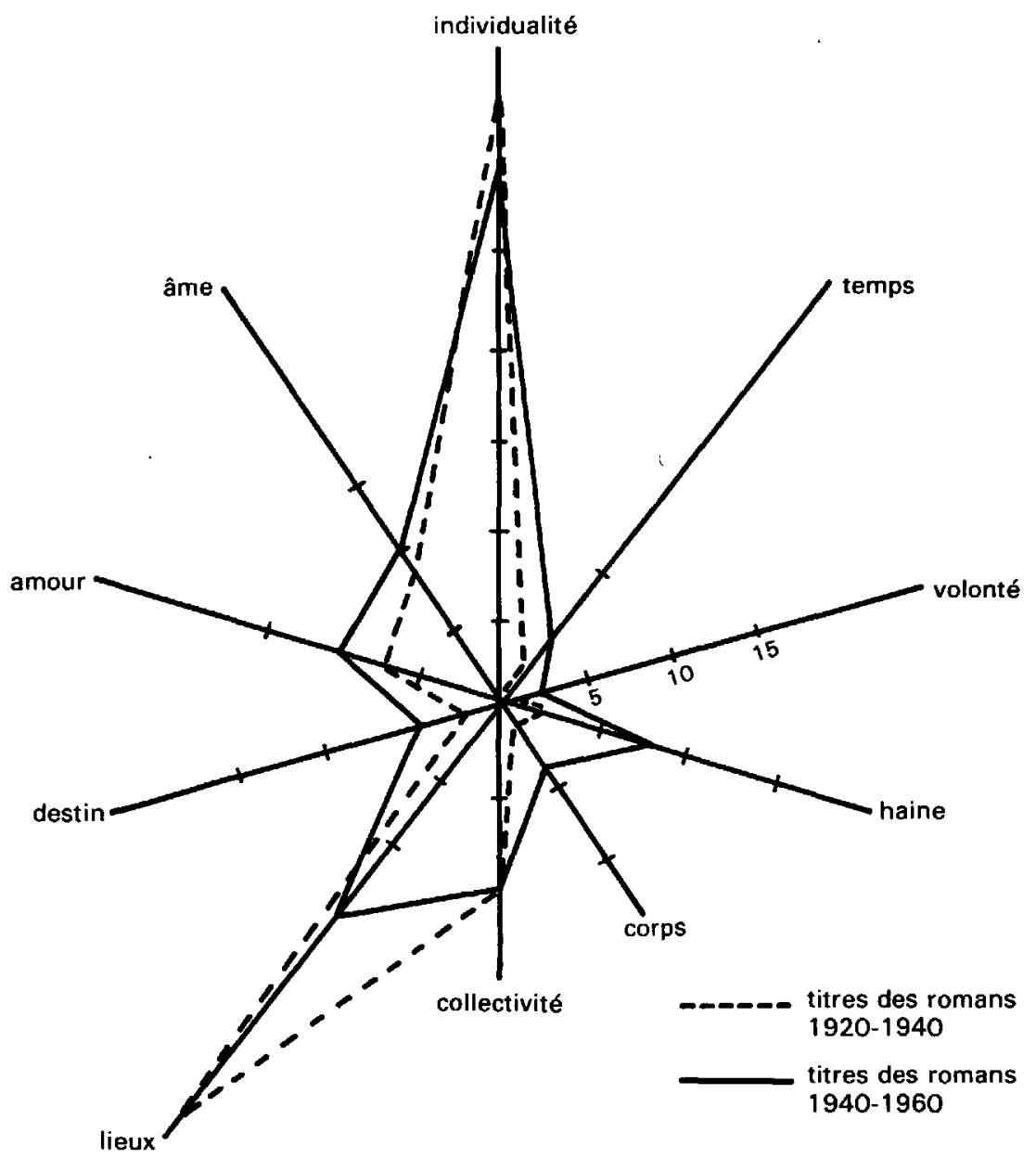

mesure qualitative des axes (en \%)

(nombre de titres ayant le thème divisé par le total) 
Les différences d'une période à l'autre sont notables avec les «axes thématiques" $3-3^{\prime}, 44^{\prime}$ et 5-5'. On peut dire que les titres de romans des années 1940 à 1960 insistent davantage sur les axes amour/haine, âme/corps et destin/volonté. Ils insistent aussi beaucoup moins sur le thème des lieux (appartenant davantage au roman du terroir). Pour ce qui est des équivalences sur l'axe 2-2' (individu-collectivité), il faut noter un fait que le graphique ne peut transmettre à cause de l'absence de la variable chronologique. En effet, l'analyse a fait ressortir, en cours de route, que ce qui fait la force de l'individualité dans la période 1920-1940, ce sont les titres parus après 1930. Ce qui, sur ce plan, remet en cause la pertinence d'établir la coupure de 1940 à 1960 plutôt que de 1930 à 1950 (puisque l'on considère 1950 comme étant l'année où le genre «roman du cas de conscience» ou «roman psychologique" s'est accaparé le monopole de la production romanesque). De toute manière, ceci indique bien que la problématique du roman était en pleine mutation dès 1930 .

On vient de voir tout au long de l'étude sémantique 22 que les titres ont $m$ is en évidence des aires sémantiques réductibles à des «substrats idéologiques » puisqu'ils sont compatibles avec l'horizon d'attente (le code dominant réglanit la lisibilité) des romans de l'époque. Mais, cette mise en évidence aurait pu être accentuée, car il est certain que des valeurs opposées (notamment entre le roman social et le roman psychologique) s'affrontent et s'annulent dans ces résultats, de sorte que les valeurs spécifiques à un groupe de producteurs (réuni autour d'un genre, donc d'un discours cristallisant des représentations en substrats) ne sont pas ressorties nettement. En fait, en termes de typologie des genres, la grande différence entre le roman psychologique (dominant) et les romans historique et social (dominés) réside (on l'aura deviné) dans la focalisation de l'axe 1-1' pour le genre psychologique. Il faut cependant mentionner que le roman social focalise de façon plus marquée l'axe 3-3'.

\section{La pragmatique}

L'analyse des rapports du signe (titre) avec ses utilisateurs (les interprétants, c'est-à-dire, ici, la critique officielle, "consacrante" ou xexcommuniante») fournit la pragmatique du signe. Celle du titre vise à rendre compte d'une certaine adéquation entre le titre d'un roman et la réception de celui-ci sur le marché littéraire. Cette sorte de vérification de la corrélation titreréception permet de déceler oú bien un fonctionnement de la critique (du comment-taire), ou bien un mécanisme interne à la codification titrologique, codification qui serait basée sur le seuil de lisibilité constituant l'horizon d'attente.

D'abord, ma première hypothèse est que le titre suscite deux centres d'intérêt. $\mathrm{Ou}$ il désigne l'événement négateur prétextant un renforcement de la positivité (c'est le désordre), ou il désigne cette positivité même (c'est l'ordre) ${ }^{23}$. Evidemment, il faut présupposer que le titre n'a d'autre possibilité 
que celle d'indiquer, par condensation ou par déplacement, un élément du texte qu'il précède. Cet élément participe à l'un ou l'autre centre d'intérêt. En nuançant davantage, on peut dire que, s'il ne désigne pas l'un des deux centres, c'est qu'il en montre leur contradiction. On peut dès lors prétendre qu'il fonctionne d'après le carré des contraintes ssémiotiques (cf. Greimas et Rastier):

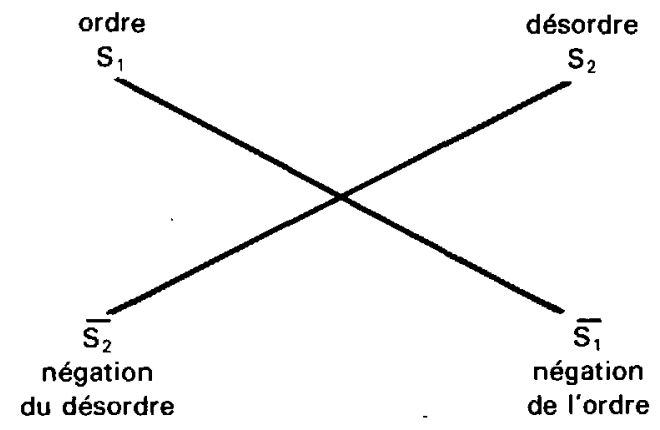

De cette manière, le manque à savoir inhérent au titre est comblé par la lecture du roman. Cependant, certains titres ne posent aucune ambiguité et désignent ouvertement (du moins en surface) l'un des quatre termes. De la sorte, le manque à savoir s'en trouve comblé dès la lecture du titre. C'est ainsi que j'ai pu établir une classification de plusieurs titres en fonction de la désignation "plus ou moins évidente» (et c'est ici qu'entre en ligne de compte l'intuition analytique) de l'ordre, du désordre ou de leur négation respective. Évidemment, une telle classification reste sujette à des remaniements ou des (ré)ajustements; je ne peux prétendre avoir été infaillible. Aussi, il n'est pas vérifié que certains éléments du titre renvoient à autre chose qu'à l'ordre ou au désordre du/dans le récit (on a qu'à penser aux titres-références culturelles cónme La Fille du roy, 1954). Une vérification radicale aurait commandé de traverser systématiquement tout le corpus.

Il est certain que la distinction entre d'une part $S_{1} / \bar{S}_{2}$ et d'autre part entre $S_{2} / \widehat{S}_{1} n$ 'est pas toujours facile à déterminer ${ }^{24}$. Par ailleurs, tous les titres n'entrant apparemment dans aucune de ces catégories ont été reportés à deux autres classes: désignation d'agents ou d'objets et désignation de données spatio-temporelles. II faudrait voir, pour leur classification dans le modèle « $S_{1}\left(\overline{S_{2}}\right)$ vs $S_{2}\left(\overline{S_{1}}\right)$, en quoi ces agents-objets ou lieux-temps entretiennent, dans le roman, un rapport avec l'ordre et le désordre. Toutefois, une hypothèse peut être posée : tous les titres comportant la désignation d'un agent, désignent probablement l'ordre du fait même que l'anti-héros n'était pas introduit dans le code romanesque de l'époque (du moins, pas systématiquement). Dès lors, le nom propre ou commun pour un agent renvoie nécessairement au héros 
sur qui repose la valorisation de l'ordre (le désordre étant: 1 . une injustice à son égard; 2. résolu par lui). Ce qui signifie qu'en ne reprenant que les titres désignant un agent-héros par un nom propre (comme Monsieur Bigras, 1944) ou par un nom commun (comme Le Survenant, 1945), il n'y a plus quarantedeux titres seulement désignant l'ordre, mais au moint cent douze. Et cela, c'est sans compter les titres désignant lieux, temps ou objets, sur lesquels je pourrais poser - avec moins d'assurance cependant - la même hypothèse. Voici quelques exemples de titres pour chacun des termes:

$\mathrm{S}_{1}$ : Les beaux jours reviendront (1940), Intimité ou Bonheur d'un jour (1946), Les Enfants qui s'aiment (1956);

$\bar{S}_{2}$ : La Revanche du destin (1943), La Fin des songes (1950);

$\mathrm{S}_{2}$ : Impasse (1946), La Bagarre (1958);

$\bar{S}_{1}$ : Autour d'un mystère (1944), Les Jours sont longs (1951).

La seconde hypothèse de travail que je pose pour rendre compte d'une pragmatique du titre se formule ainsi : est bien reçu tout roman dont le titre renvoie aux termes de la déixis positive $\left(S_{1}\right.$ et $\left.\bar{S}_{2}\right)$ et mal reçu tout roman dont le titre renvoie aux termes de la déixis négative $\left(S_{2}\right.$ et $\left.\bar{S}_{1}\right)$. II restait à vérifier la validité de cette hypothèse dans la classification établie. Pour mener à terme cette tâche, j"ai constitué un échantillon de vingt-six titres (soit dix pour cent du corpus) et je suis allé lire un compte rendu officiel de l'époque (presque toujours dans la revue Lectures, médiatrice entre la production et le public) pour examiner deux choses: d'abord ce que désignait le titre (le compte rendu donnant souvent un bref résumé); ensuite, la réception qu'on accordait au roman.

Ce travail a permis de repérer le fonctionnement transformationnel du rapport entre texte et titre. On considère que le titre d'un roman non lu constitue la structure de surface du texte: c'est le "signifiant". La lecture entraîne des transformations qui permettent de retracer la structure profonde, donc le «signifié» du titre. II existe, selon Hoek ${ }^{25}$, six transformations:

1. la transformation zéro $\left(T_{1}\right)$ qui transforme le sème du titre (s) en le même sème placé dans le contexte narratif (S). Cette transformation $\left(s_{1} \longrightarrow S_{1}\right)$ rend bien compte de la fonction d'anticipation où la lecture ne fait que dévoiler l'adéquation du titre au texte:

2. la transformation de conjonction $\left(T_{2}: s_{1} \vee S_{2} \rightarrow S_{1} \wedge S_{2}\right)$ qui joint dans le texte deux ou plusieurs sens initialement disjoints dans le titre. Exemple emprunté à Hoek: La Jalousie (de Robbe-Grillet) où $s_{1}$ correspond au sentiment d'envie et $s_{2}$ à la persienne;

3. la transformation d'addition $\left(T_{3}: s_{1} \longrightarrow S_{1}+S_{2}\right)$ qui ajoute un ou plusieurs sens au premier sens du titre;

4. la transformation de substitution $\left(T_{4}: S_{1} \longrightarrow S_{2}\right)$ qui rend compte des 
titres métaphoriques. Ce qui distingue $T_{4}$ de $T_{3}$, c'est que le second sens rend, en $\mathrm{T}_{4}$, le premier superflu;

5. la transformation de disjonction $\left(T_{5}: S_{1} \vee S_{2} \longrightarrow S_{1} \wedge S_{2}\right)$ où l'ensemble des sens suggérés par le titre devient incompatible avec le contexte narratif. Exemple emprunté à Hoek: L'Amante anglaise (Duras) devient "la menthe anglaise";

6. les transformations mixtes $\left(T_{6}\right)$ constituant une combinaison de deux transformations (le plus souvent $T_{2}$ et $T_{3}$ ).

Mais, un phénomène fort intéressant (trop peut-être) est apparu lors de la vérification de ces modèles (réception et transformations) sur le corpus 40-60. En effet, il faut signaler trois choses: 1) deux transformations en particulier sont généralement utilisées (dans quatre-vingt pour cent des cas): il s'agit surtout de $T_{1}$ et quelquefois de $T_{4} ; 2$ ) lès titres désignant un terme de la déixis positive $\left(S_{1}\right.$ ou $S_{2}$ ) appartiennent à des textes qui ont eu droit à des commentaires favorables de la part de la critique; en revanche, les textes de Giroux et Langevin (Le Gouffre a toujours soif et Poussiere sur la ville) classés sur la déixis négative $\left(\bar{S}_{1}\right.$ et $S_{2}$ respectivement) n'ont pas obtenu une excellente réception contrairement à la réputation dont ils jouissent dans nos manuels scolaires ${ }^{26}$. Ce qui confirme, aussi bien par la positive que par l'absurde, mon hypothèse sur la pragmatique (à savoir: est bien reçu tout texte dont le titre désigne $S_{1}$ ou $\left.\widehat{S}_{2}\right)$; là où ne se vérifie plus l'hypothèse (15\% des cas), il s'agit de titres où la lecture effectue une transformation autre que $T_{1}\left(T_{3}, T_{4}, T_{5}\right)$ :

a) Risques d'hommes, $1950\left(\mathrm{~T}_{3}\right)$ : classé en $\mathrm{S}_{2}$, mais réception positive;

b) Le Poids du jour, $1949\left(\mathrm{~T}_{4}\right)$ : classé en $\mathrm{S}_{2}$, mais réception positive;

c) Pacte et Complot, $1955\left(\mathrm{~T}_{5}\right)$ : classé en $\mathrm{S}_{1}+\mathrm{S}_{2}$ (ambiguïté), mais réception négative (on ne peut supposer ici que $S_{2}$ finit par prédominer sur $S_{1}$, sinon comment expliquer que pour un titre comme L'Amour et l'Épreuve, 1940, classé également en $S_{1}+S_{2}$, c'est le phénomène inverse qui s'est produit?)

Cette situation peut signifier que l'hypothèse ne tient que lorsqu'il y a transformation zéro $\left(T_{1}\right)$. Ce qui amène à poser des considérations générales qui appellent des vérifications pour confirmer ou infirmer cette pragmatique du titre: pour constituer une pragmatique du titre (du moins celle dont je cherche à rendre compte), il faut tout d'abord vérifier quelle est ou quelles sont les transformations les plus utilisées dans le corpus (constitution d'un code transformationnel du titre à une époque donnée); ensuite, en fonction de ce code transformationnel, on peut "prédire» la réception du texte compte tenu de l'horizon d'attente de l'époque concernée. Sur le corpus 1940-1960, la «prédiction» devenait facile à cause de l'utilisation presque généralisée de la $T_{1}$. Sur d'autres corpus, où ce seraient d'autres transformations qui auraient été valorisées (codées) par la norme en place (dominante), il faudrait examiner si la corrélation titre/réception est aussi puissante ${ }^{27}$. 
On peut interpréter la situation autrement: à transformation zéro du titre, on obtient réception conforme du texte. On décèle là une fonction primordiale attribuée au titre à l'époque auprès des lecteurs: rendre compte de ce qui est dit dans le roman. Mais aussi, fonction occultant le travail idéologique du texte-titre résidant dans le renforcement naturalisé d'une positivité par le prétexte d'une négativité temporaire. Le titre est ainsi «une des ruses du texte qui ont pour fonction d'imprégner le lecteur d'une morale, d'une idéologie [...] et cette ruse est d'autant plus dangereuse qu'on s'en aperçoit.moins ${ }^{28}$ ”.

\section{CONCLUSION}

Sans prétendre avoir vidé la question du titre dans la production québécoise des années 1940-1960, on a vu que celui-ci identifie le texte en désignant certaines valeurs (type d'information, thèmes, ordre/désordre) qui sont souvent propres à rendre compte de sa réception. Donc, l'intention du texte, qu'affiche en quelque sorte le titre, peut être utilisée dans une approche sociologique de la réception des produits littéraires.

La syntaxe a finalement démontré, à un faible niveau il est vrai, qu'une combinatoire de différences syntaxiques permettait d'instituer un code titrologique propre à chaque genre. Un résultat similaire a été obtenu à partir de l'analyse sémantique. Les trois principaux genres en concurrence à l'époque (romans historique, social et psychologique ou cas de conscience) employaient déjà dans le titre un code de différenciation. Et comme le roman psychologique a pu établir une certaine cristallisation de sa dominance sur le marché autour de 1950 , les romans historique et social ont dû s'effacer devant les positions dominantes dans le champ littéraire. II s'agirait, à ce stade-ci de la recherche titrologique, d'examiner de quelle manière cela se concrétise au niveau de la codification des titres ${ }^{29}$. Car, si «le titre propose un certain nombre de sens plus ou moins définis et qui sont entièrement ou partiellement mis en valeur par le texte ${ }^{30} \%$, cette mise en valeur propose une certaine qualité de la réception en fonction d'une conformité entre cette même mise en valeur et l'horizon d'attente d'une époque.

Mais, naturellement, il reste à développer des modèles suffisamment adéquats, sans être trop puissants, pour rendre compte d'une pragmatique du titre. Ce que j'ai suggéré ici, de manière trop partielle et trop hypothétique, appelle des vérifications empiriques sur d'autres corpus, moins longs mais plus fouillés.

De plus, en termes de théorie du titre, il y aurait matière à élaborer la remarque de la note 12 . Si le titre est condensation, déplacement, fragment(s) du texte, il y a là de quoi supposer qu'il fonctionne (structurellement) comme retour du refoulé, comme fantasme du texte qu'il précède. D'autant plus qu'il en est l'inscription. Inscription du désir d'une lecture dans (et par) une histoire (réelle et fictive) du Texte. Il s'agit moins de voir de quoi il va s'agir que d'où ca va agir. 
Or, beaucoup de travail reste à accomplir... Mais, il faut bien que l'un annonce pour que l'autre explique. C'est bien là titrer...!

1. Pour les deux citations: Duchet, 1973, p. 50.

2. Hoek, 1973, p. 3.

3. Exemple emprunté à Hoek, 1973, p. 2-4.

4. Grivel, 1973a, respectivement pour les deux citations, p. 168 et 166.

5. Duchet, 1973, p. 57.

6. Pour plus de précisions sur l'horizon d'attente de l'époque, voir les travaux de Jacques Michon, Hélène Lafrance et Richard Berger dans Structure, Idéologie et Réception du roman québécois de 1940-1960. Sherbrooke, document de travail FCAC (études françaises), Université de Sherbrooke, 1979, 108 pages.

7. A ma connaissance, il s'agit là ḍ̛ un précédent.

8. Grivel, 1973a, p. 166-168.

9. Hoek, 1973, p. 4

10. Hoek, 1972, p. 291.

11. Hoek, 1973, p. 7 et 1972, p. $290-291$.

12. Avec une telle terminologie, on croirait bien que le titre est le fantasme du texte: à développer.

13. Duchet, 1973, p. 52.

14. Grivel, 1973a, p. 172.

15. Cf. «Rhétorique de $»$ in Communications $n^{\circ} 4,1964$.

16. Grivel, 1973b, p. 143-183.

17. Hoek, 1973 , p. $30-44$ et 1974, p. 112-117.

18. Pour donner un exemple de la surdétermination du code, je peux procéder par la négative en indiquant certains titres de romans échappant au code titrologique du genre dans la mesure où ils peuvent appartenir à un autre genre lou plus justement: macro-genre). C'est ainsi que Profil de l'orignal (1952) ou La Louve (1952) ou même encore $C e$ que peut l'amour (1943) peuvent être lus comme titres d'ouvrages documentaires (à noter l'importance de la construction syntaxique: «Profil d'un orignal» aurait éliminé le générique conditionnant le code du titre de documents non fictionnels ou spécialisés).

19. Voir note 6.

20. Un tel pléonasme fait songer à la possibilité d'établir une «rhétorique des titres».

21. Voir note 6.

22. Pour l'étude sémantique, on pourrait me reprocher de n'avoir tenu compte que de la dénotation des titres. Le problème de la connotation ou d'une signification autre (comme par exemple: Et le septième jour. 1958, référant à la mythologie chrétienne) dans les titres devient difficile à analyser vue l'imprécision opératoire qui entoure déjà le concept de connotation. Mais, ici aussi, une piste de recherche devrait s'ouvrir.

23. En ce qui a trait à cette conception de désordre renforçant l'ordre, cf. Grivel, 1973a, p. 138-152.

24. Comme on me l'a fait remarquer, il s'agit ici d'une utilisation insuffisante du carré des contraintes sémiotiques. La simple dichotomie "affirmation de l'ordre vs négation de l'ordre» aurait pu être opératoire. Cependant, je signale au passage que les titres situés sur les pôles contradictoires $\left(\bar{S}_{1}\right.$ et $\left.\bar{S}_{2}\right)$ ont reçu des critiques plus ambiguës, alors que, souvent, les titres situés sur $S_{1}$ et $S_{2}$ étaient beaucoup plus clairs quant à la consécration ou à l'excommunion : on consacrait (pour $S_{1}$ ) ou rejetait (pour $\mathbf{S}_{2}$, quand il y avait critique, ce qui n'était pas toujours le cas) le roman. 
25. Hoek, 1972, p. 300-302.

26. II ne faut pas oublier que les manuels scolaires sélectionnent les ceuvres depuis leur époque. C'est dire que l'horizon d'attente de ces manuels diffère de celui régnant lors de la parution des textes.

27. Pour le corpus $40-60$, la corrélation statistique a été très puissante: +.85 , quand on pense que la borne inférieure est -1 et la borne supérieure est +1 .

28. Hoek, 1973, p. 10.

29. II ne faudrait pas non plus oublier le genre populaire.

30. Hoek, 1974, p. 112. 


\section{BIBLIOGRAPHIE}

1. DUCHET, Claude

1973 "'La Fille abandonnée' et 'La Bête humaine', éléments de tritrologie romanesque" in Littérature, $n^{\circ} 12$ (décembre 1973), pp. 49-73.

2. GRIVEL, Charles

1973a "Puissance du titre. Sémioloaie du titre. Rèales de la titraison romanesque» in Production de l'intérêt romanesque, LaHaye-Paris, Mouton, 1973, pp. 166-181.

1973b «Tableau XIII: les sèmes du titre; les opérateurs du titre; les opérations du titre " in Production de l'intérêt romanesque, volume complémentaire, Amstelveen, Hoekstra offset, 1973, pp. 143-187.

3. HOEK, Léo

1972 «Description d'un archonte: préliminaires à une théorie du titre à partir du Nouveau Roman" in Nouveau Roman : hier, aujourd'hui, tome I, Paris, UGG, «10/18», 1972, pp. 289-306.

1973 "Pour une sémiotique du titre" in Documents de travail et prépublications du Centro Internaltionale di Semiotica e di Linguistica, nos 20-21, Universita di Urbino, (janvier-février 1973), pp. 1-52.

1974 «L'imposture du titre ou la fausse vraisemblance» in C. Grivel, et A. K. Varga, Du linguistique au textuel, Van Gorcum, Assen/Amsterdam, 1974, pp. 111-120. 